\title{
Measurement of the Running of the Electromagnetic Coupling at LEP
}

\author{
Salvatore Mele \\ INFN, Sezione di Napoli, I-80125, Napoli, Italy \\ Salvatore.Mele@cern.ch
}

\begin{abstract}
The study of low-angle and large-angle Bhabha scattering at LEP gives access to the running of the electromagnetic coupling. Two recent measurements of the OPAL and L3 collaborations probe the running of $\alpha$ in the regions $1.8 \mathrm{GeV}^{2}<-Q^{2}<$ $6.1 \mathrm{GeV}^{2}$ and $1800 \mathrm{GeV}^{2}<-Q^{2}<21600 \mathrm{GeV}^{2}$, respectively. The strategies and the results of these studies are presented. A global overview is given of the agreement of these and previous L3 findings with the QED predictions.
\end{abstract}

To appear in the Proceedings of the International Europhysics Conference on High Energy Physics July 21st - 27th 2005, Lisboa, Portugal 


\section{Introduction}

A remarkable feature of quantum field theory is the dependence of coupling constants on the energy scale of the processes in which their corresponding interactions occur. In QED, large momentum-transfers probe virtual-loop corrections to the photon propagator, as sketched in Figure 1, inducing a dependence of the electromagnetic coupling, $\alpha$, on the squared momentum transfer, $Q^{2}$. This evolution, or running, of $\alpha$ is parametrised as [1]:

$$
\alpha\left(Q^{2}\right)=\frac{\alpha_{0}}{1-\Delta \alpha\left(Q^{2}\right)}
$$

where $\alpha_{0}$ is the fine-structure constant. This is measured with high accuracy in solid-state processes and via the study of the anomalous magnetic moment of the electron to be $1 / \alpha_{0}=137.03599911 \pm 0.00000046$ [2]. The contributions to $\Delta \alpha\left(Q^{2}\right)$ from lepton loops are precisely predicted, while those from quark loops are difficult to calculate due to non-perturbative QCD effects. They are estimated using dispersion-integral techniques. At the scale of the Z-boson mass, a recent calculation yields $\alpha^{-1}\left(m_{\mathrm{Z}}^{2}\right)=128.936 \pm 0.046$ [3].

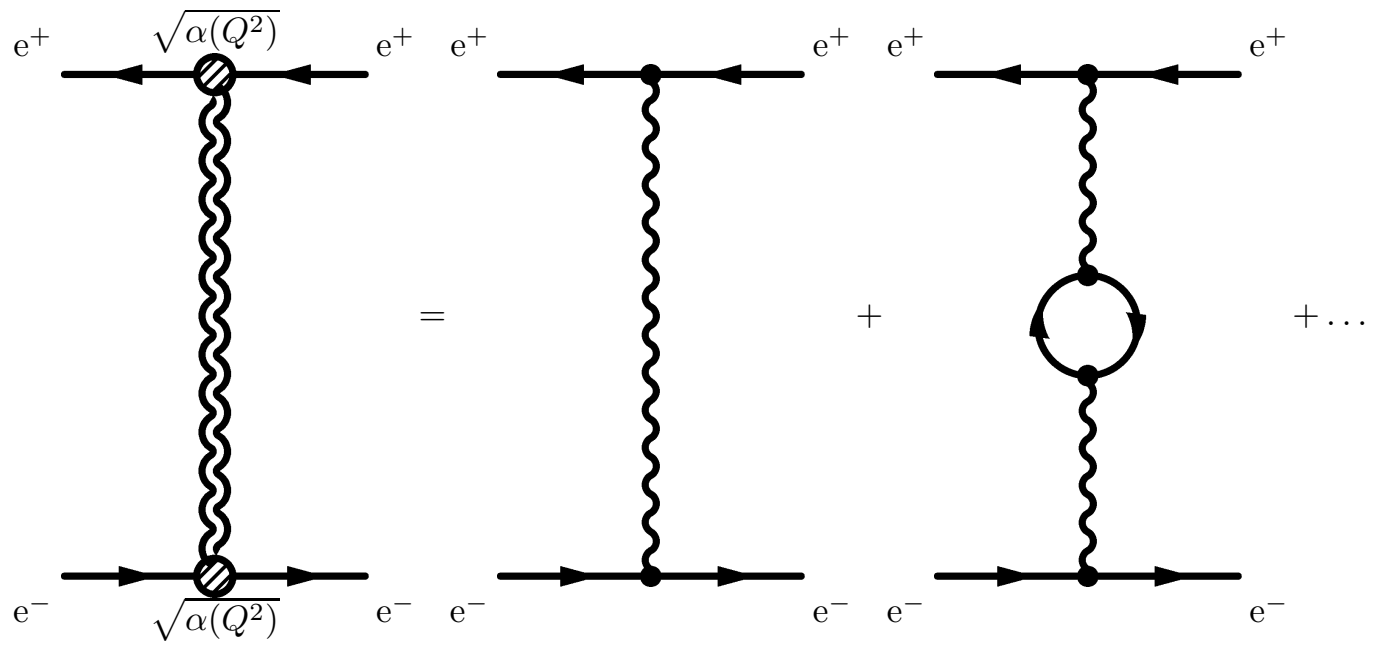

Figure 1: t-channel Feynman diagrams contributing to Bhabha scattering and the phenomenon of vacuum-polarisation. The sum of all diagrams including zero, one, two or more vacuum-polarisation insertions is denoted by the diagram to the left with the double-wavy photon propagator, with an electromagnetic coupling $\alpha\left(Q^{2}\right)$.

Bhabha scattering at $\mathrm{e}^{+} \mathrm{e}^{-}$colliders, $\mathrm{e}^{+} \mathrm{e}^{-} \rightarrow \mathrm{e}^{+} \mathrm{e}^{-}$, constitutes a unique window on the vacuum-polarisation insertions of Figure 1. Its measurement 
gives access to the running of $\alpha$ in the space-like region, $Q^{2}<0$. The fourmomentum transfer in Bhabha scattering is measured with high precision through its dependence on the squared centre-of-mass energy, $s$, and on the scattering angle, $\theta: Q^{2}=t \simeq-s(1-\cos \theta) / 2$. Low-angle, $\theta \sim 1^{\circ}-3^{\circ}$, and large-angle, $\theta \sim 20^{\circ}-90^{\circ}$, Bhabha scattering correspond to the low- $Q^{2}$ and high- $Q^{2}$ regimes, respectively.

A caveat is in order when discussing the extraction of information on the running of $\alpha$ from Bhabha scattering. The cross section of this process can be written as:

$$
\begin{gathered}
\frac{\mathrm{d} \sigma}{\mathrm{d} t}=\frac{\mathrm{d} \sigma^{(0)}}{\mathrm{d} t}\left(\frac{\alpha(t)}{\alpha_{0}}\right)^{2}(1+\varepsilon)\left(1+\delta_{\gamma}\right)+\delta_{\mathrm{Z}}, \\
\text { with tree }- \text { level cross section } \frac{\mathrm{d} \sigma^{(0)}}{\mathrm{d} t}=\frac{4 \pi \alpha_{0}^{2}}{t^{2}} .
\end{gathered}
$$

Naively, one could imagine inserting the measured cross section in the lefthand side of Equation 2, and incorporate the knowledge of the $s$-channel contributions, $\delta_{\gamma}$ and $\delta_{\mathrm{Z}}$, and of the radiative correction, $\varepsilon$, to extract a value for $\alpha(t)$. This argument is unfortunately flawed as the measurement of the cross section requires knowledge of the integrated luminosity. At LEP this is estimated by measuring events from low-angle Bhabha scattering and assuming its cross section which, in turns, depends on $\alpha(t)$. Therefore, the only information which is experimentally accessible concerns the evolution of $\alpha$ over a $Q^{2}$ range. Two recent measurements of the running of $\alpha$ are discussed in the following, along with some previous measurements, and, finally, a combined overview.

\section{Previous measurements}

The L3 collaboration first established the running of $\alpha$ in the range $2.1 \mathrm{GeV}^{2}<$ $-Q^{2}<6.2 \mathrm{GeV}^{2}[4]$ by comparing event counts from low-angle Bhabha scattering in different regions of its luminosity monitor, with a result:

$$
\alpha^{-1}\left(-2.1 \mathrm{GeV}^{2}\right)-\alpha^{-1}\left(-6.2 \mathrm{GeV}^{2}\right)=0.78 \pm 0.26,
$$

where the uncertainty combines statistical and systematic uncertainties.

The running of $\alpha$ in large-angle Bhabha scattering was first investigated by the VENUS Collaboration at TRISTAN in the range $100 \mathrm{GeV}^{2}<-Q^{2}<$ $2916 \mathrm{GeV}^{2}$ [5]. Later, the L3 Collaboration studied the same process at $\sqrt{s}=$ $189 \mathrm{GeV}$ for scattering angles $20^{\circ}-36^{\circ}$, probing the range $12.25 \mathrm{GeV}^{2}<$ $-Q^{2}<3434 \mathrm{GeV}^{2}[4]$, finding:

$$
\alpha^{-1}\left(-12.25 \mathrm{GeV}^{2}\right)-\alpha^{-1}\left(-3434 \mathrm{GeV}^{2}\right)=3.80 \pm 1.29,
$$


where the uncertainty comprises statistical and systematic sources.

\section{Precision measurement at low $Q^{2}$}

The OPAL collaboration recently performed a high-precision study of the running of $\alpha$ at low- $Q^{2}[6]$ using data collected with their luminometer. This consisted of tungsten absorber and 32-pad silicon detectors, covering a polar angle $1.4^{\circ}-3.3^{\circ}$. The analysis relies on 10 million high-energy back-to-back cluster pairs originating from low-angle Bhabha scattering at $\sqrt{s}=m_{\mathrm{Z}}$, corresponding to a momentum transfer $1.8 \mathrm{GeV}^{2}<-Q^{2}<6.1 \mathrm{GeV}^{2}$. The $t$ spectrum of the events is investigated to extract information on $\alpha(t)$. Data are divided in five $t$ bins and compared with the BHLUMI Monte Carlo [7], as shown in Figure 2a. The ratio between data and Monte Carlo counts for the hypothesis $\alpha(t)=\alpha_{0}$ is fitted with the function $a+b \ln \left(t / t_{0}\right)$, where $t_{0}=-3.3 \mathrm{GeV}^{2}$ is the mean value of $t$ in the data sample and the parameter $b$ is related to the running of $\alpha$ as

$$
\Delta \alpha\left(t_{2}\right)-\Delta \alpha\left(t_{1}\right) \approx \frac{b}{2} \ln \frac{t_{2}}{t_{1}} .
$$

Three hypotheses are considered and found to be completely excluded by the data: $\alpha(t)=\alpha_{0}$; a running of $\alpha$ induced only by electron virtual-loops; a running of $\alpha$ induced only by lepton virtual-loops. The QED hypothesis of a running with virtual loops of both leptons and quarks fits the data best. The result of a fit for $b$, through Equation 5, yields:

$$
\Delta \alpha\left(-6.1 \mathrm{GeV}^{2}\right)-\Delta \alpha\left(-1.8 \mathrm{GeV}^{2}\right)=(440 \pm 58 \pm 43 \pm 30) \times 10^{-5},
$$

in excellent agreement with the QED prediction of $460 \times 10^{-5}$. The first

uncertainty is statistical, the second systematic -dominated by the simulation of the detector material and by the reconstruction of the radial coordinate of the clusters- and the third theoretical.

This measurement proves the running of $\alpha$ at low- $Q^{2}$ with a significance of $5.6 \sigma$, and, for the first time, establishes the hadronic contribution to the running with a significance of $3.0 \sigma$ as:

$$
\Delta \alpha_{\text {had }}\left(-6.07 \mathrm{GeV}^{2}\right)-\Delta \alpha_{\text {had }}\left(-1.81 \mathrm{GeV}^{2}\right)=(237 \pm 58 \pm 43 \pm 30) \times 10^{-5} \text {. }
$$

\section{$4 \quad$ First measurement at large $Q^{2}$}

The L3 collaboration measured the differential cross section for Bhabha scattering for scattering angles in the range $26^{\circ}-90^{\circ}$. About 40000 events are 

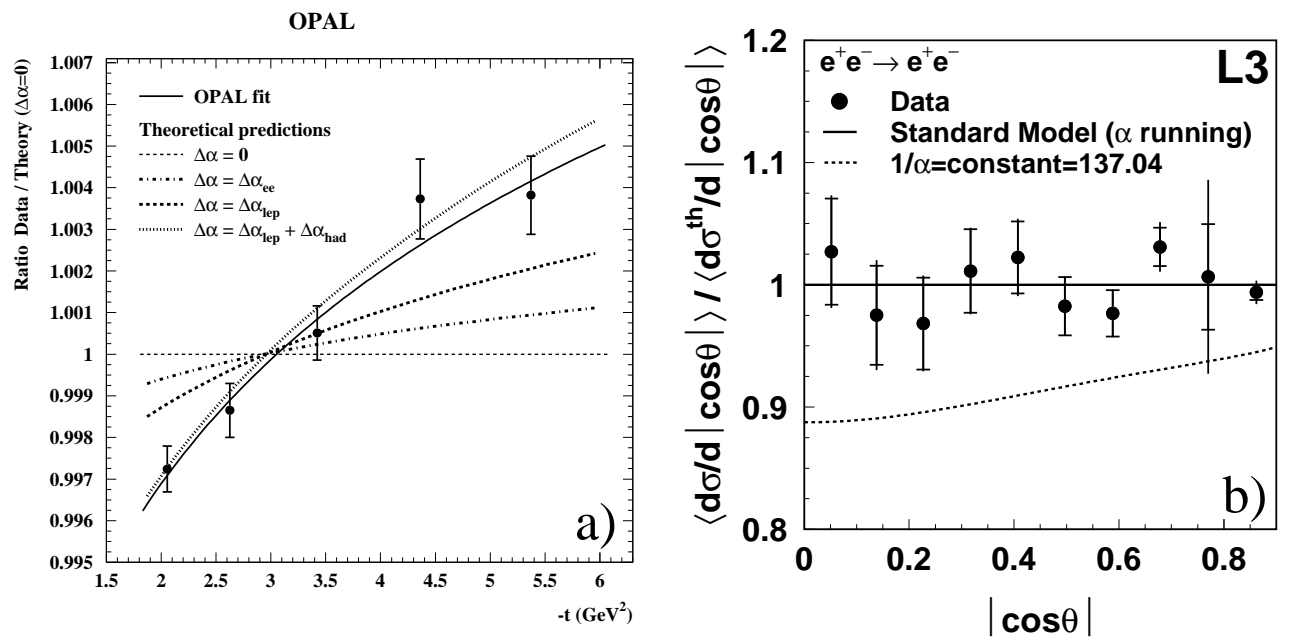

Figure 2: Experimental results proving the running of $\alpha$ at a) low and b) high $Q^{2}$. a) The ratio of event counts in different radial regions of the OPAL luminometer, corresponding to different values of $t$, for data and a Monte Carlo simulation with $\alpha(t)=\alpha_{0}$, from Reference [6]. The data favour the QED prediction of $\Delta \alpha(t)$ and exclude all other scenarios. Only statistical uncertainties are shown. b) The ratio of centre-of-mass-averaged differential cross section of for large-angle Bhabha scattering measured by L3 divided by the theoretical predictions, from Reference [9]. The inner error bars denote the statistical uncertainties, the outer the combination of statistical and systematic uncertainties. The data exclude the scenario $\alpha(t)=\alpha_{0}$. 
selected as back-to-back clusters in the high-resolution BGO electromagnetic calorimeter with matched tracks, at $\sqrt{s}=109-209 \mathrm{GeV}$, corresponding to $1800 \mathrm{GeV}^{2}<-Q^{2}<21600 \mathrm{GeV}^{2}$. The 80 measured values of the cross section, for each of ten angular ranges and eight centre-of-mass energies, are compared with the predictions of the BHWIDE Monte Carlo [8] to extract information on the running of $\alpha$ [9]. Figure 2b compares the data and the predictions for the centre-of-mass-averaged cross sections. The running of $\alpha$ is parametrised as:

$$
\alpha\left(Q^{2}\right)=\frac{\alpha_{0}}{1-C \Delta \alpha\left(Q^{2}\right)} .
$$

The hypothesis $\alpha\left(Q^{2}\right)=\alpha_{0}$, corresponding to $C=0$, is completely excluded, whereas the data are in excellent agreement with the running predicted in QED, corresponding to $C=1$. A fit determines

$$
C=1.05 \pm 0.07 \pm 0.14
$$

where the first uncertainty is statistical and the second systematic, dominated by theoretical uncertainties with some contribution from the detector modelling.

\section{$5 \quad$ Two textbook plots?}

Figures $3 \mathrm{a}$ and $3 \mathrm{~b}$ summarise the LEP results on the running of the electromagnetic coupling. In both figures, the L3 measurement at $1800 \mathrm{GeV}^{2}<$ $-Q^{2}<21600 \mathrm{GeV}^{2}[9]$ is represented as a yellow band, obtained by inserting the measured value of $C$ from Equation 9 into Equation 8 and assuming the QED description of $\Delta \alpha\left(Q^{2}\right)$ of Reference [3].

In Figure 3a, the OPAL measurement at $1.8 \mathrm{GeV}^{2}<-Q^{2}<6.1 \mathrm{GeV}^{2}$, Equation 6, and the L3 measurements at $2.1 \mathrm{GeV}^{2}<-Q^{2}<6.2 \mathrm{GeV}^{2}$ and $12.25 \mathrm{GeV}^{2}<-Q^{2}<3434 \mathrm{GeV}^{2}$, Equations 3 and 4 , respectively, are represented with a pair of symbols each. The empty symbol represents the value of $\alpha^{-1}\left(Q^{2}\right)$ fixed at the lower end of each $Q^{2}$ range with Equation 1 and the QED description of $\Delta \alpha\left(Q^{2}\right)$ of Reference [3]. The full symbol represents the values of $\alpha^{-1}\left(Q^{2}\right)$ at the higher end of each $Q^{2}$ range extracted from these fixed values and the measurements in Equations 6, 3 and 4.

Figure $3 \mathrm{~b}$ goes one step further, anchoring the lower end of each $Q^{2}$ range by using the L3 measurement of $C$ at $1800 \mathrm{GeV}^{2}<-Q^{2}<21600 \mathrm{GeV}^{2}$ and assuming it also describes the running of $\alpha$ for lower values of $Q^{2}$. First, the L3 measurement at $2.1 \mathrm{GeV}^{2}<-Q^{2}<6.2 \mathrm{GeV}^{2}$ and the OPAL measurement at $1.8 \mathrm{GeV}^{2}<-Q^{2}<6.1 \mathrm{GeV}^{2}$ are combined in a single measurement: $\alpha\left(6.1 \mathrm{GeV}^{2}\right)-\alpha\left(1.8 \mathrm{GeV}^{2}\right)=(363 \pm 52) \times 10^{-7}$. The value of $\alpha\left(1.8 \mathrm{GeV}^{2}\right)$ is 
then fixed by using the measured value of $C$ from Equation 9, the evolution expected from Equation 8 and the QED description of $\Delta \alpha\left(Q^{2}\right)$ of Reference [3]. Finally, the value of $\alpha\left(6.1 \mathrm{GeV}^{2}\right)$ is extracted by using the fixed value of $\alpha\left(1.8 \mathrm{GeV}^{2}\right)$, with an additional uncertainty which follows from the $14 \%$ uncertainty on $C$. A similar procedure is followed to extract the value of $\alpha\left(-3434 \mathrm{GeV}^{2}\right)$ from Equation 4 .

Both Figures present an excellent agreement with the QED predictions of Reference [3], represented by the solid line: two textbook plots!

\section{References}

[1] E.C.G. Stückelberg and A. Petermann, Helv. Phys. Acta 26 (1953) 499; M. Gell-Mann and F. Low, Phys. Rev. 95 (1954) 1300; N.N. Bogoliubov and D.V. Shirkov, Dokl. AN SSSR 103 (1955) 203.

[2] CODATA Task Group, Rev. Mod. Phys. 72 (2000) 351; http://physics.nist.gov/constants.

[3] H. Burkhardt and B. Pietrzyk, Phys. Lett. B 513 (2001) 46.

[4] L3 Collab., M. Acciarri et al., Phys. Lett. B 476 (2000) 40.

[5] VENUS Collab., S. Odaka et al., Phys. Rev. Lett. 81 (1998) 2428; VENUS Collab., T. Arima et al., Phys. Rev. D 55 (1997) 19.

[6] OPAL Collab. G. Abbiendi et al., Eur. Phys. J. C45 (2006) 1.

[7] S. Jadach et al., Comput. Phys. Commun. 102 (1997) 229.

[8] S. Jadach, W. Placzek and B.F.L. Ward, Phys. Lett. B 390 (1997) 298.

[9] L3 Collab., P. Achard et al., Phys. Lett. B 623 (2005) 26. 

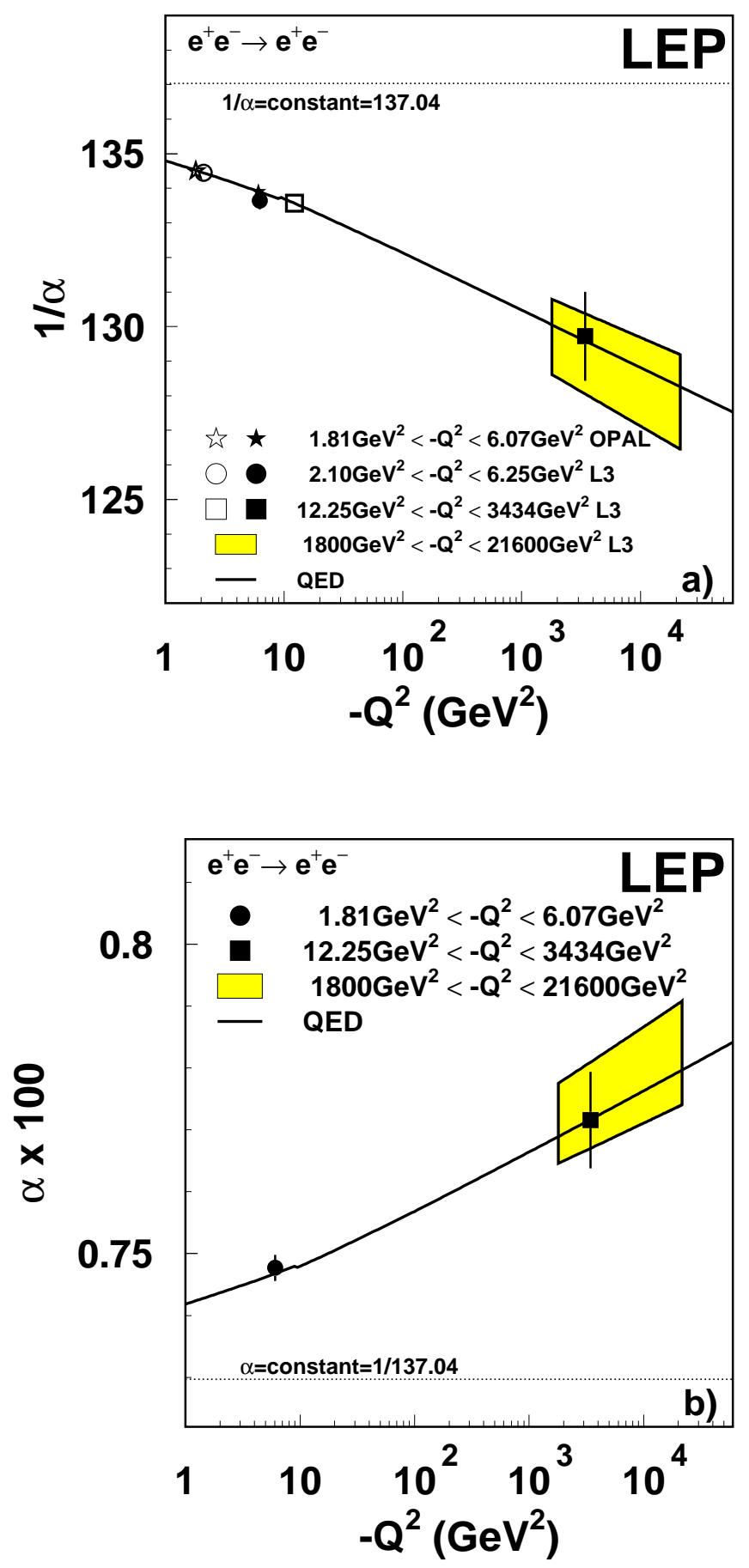

Figure 3: Comparison of LEP results on the measurement of the running of the electromagnetic coupling with QED predictions. The treatment of data and the meaning of the symbols is discussed in the last section of the text. 